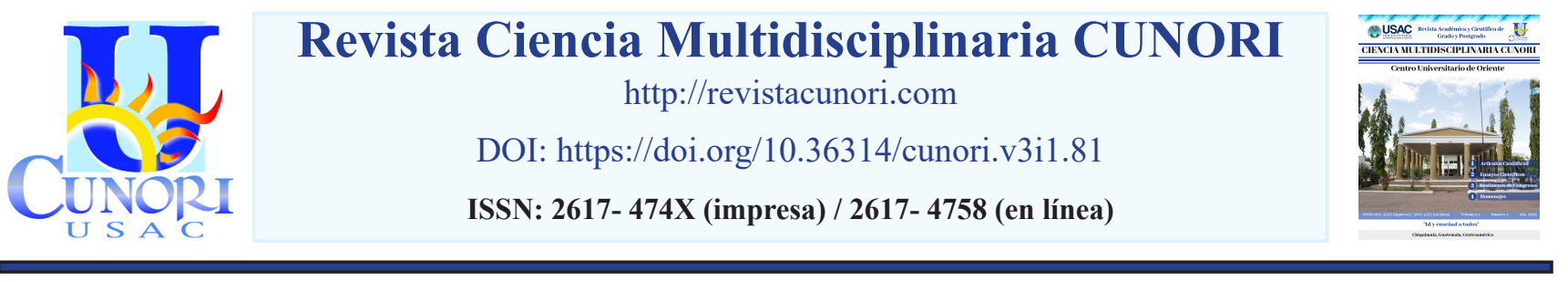

Como citar el artículo

Morales, D., Icaza, G., Arriola, C., Mazariegos, E., (2019). Recurrencia de hernias inguinales en el Hospital de Chiquimula. Revista Ciencia Multidisciplinaria CUNORI, 3(1), 65-73. DOI: https://doi.org/10.36314/cunori.v3i1.81

\title{
Recurrencia de hernias inguinales en el Hospital de Chiquimula
}

\author{
Recurrence of inguinal hernias in the Chiquimula Hospital \\ Danna Morales*, Graciela Icaza, Carlos Arriola, Edvin Mazariegos \\ Centro Universitario de Oriente (CUNORI), Universidad de San Carlos de Guatemala
}

Recibido: 05 de agosto de 2018 / Revisión: 20 de septiembre de 2018 / Aceptado: 19 de enero de 2019

Disponible en internet el 30 de agosto de 2019

\author{
*Autor para correspondencia. \\ Correo electrónico: danabymorales@hotmail.com \\ Resumen
}

Ce denomina recurrencia o recidiva al desarrollo de una nueva hernia independientemente del sitio anatómico, con la única $\checkmark$ condición de que sea del mismo lado operado previamente. El estudio realizado fue de tipo descriptivo retrospectivo, con el objetivo de determinar la recurrencia de las hernias inguinales de acuerdo a las técnicas de reparación quirúrgica en los pacientes postoperados en el Servicio de Cirugía General de adultos del Hospital Nacional de Chiquimula de enero de 2013 a diciembre de 2017. La información obtenida fue por medio de la ficha recolección datos, que ayudó a determinar una alta recurrencia de hernia inguinal con un $8 \%$ de 59 casos que fueron intervenidos por hernia inguinal, el 100\% corresponden al sexo masculino, la edad dominante fue rango de 31 a 59 años con el 60\% (3); según su cronología tiene predominio en un 80\% (4) para recurrencia tardía. La técnica más utilizada en la primera intervención quirúrgica de pacientes con hernia inguinal fue un 64\% (38) la técnica de Lichtenstein y la más utilizada en la primera intervención quirúrgica de hernia inguinal recurrente incluye otros con 60\% (3). Se socializaron los resultados obtenidos con los cirujanos, estudiantes de medicina, la Dirección del Hospital Nacional de Chiquimula y pacientes ingresados postoperados de hernia inguinal.

Palabras clave: hernia inguinal, recurrencia de hernia inguinal, técnica quirúrgica

\section{Abstract}

$\mathrm{R}$ ecurrence or recurrence is currently called the development of a new hernia regardless of the anatomical site, with the only condition that it be from the same side previously operated. The study carried out was of a retrospective descriptive type, with the objective of determining the recurrence of inguinal hernias according to the surgical repair techniques in the postoperative patients in the General Surgery Service of adults of the Hospital Nacional de Chiquimula from January 2013 to December 2017. The information obtained was by means of the data collection form, which helped to determine a high recurrence of inguinal hernia with $8 \%$ of 59 cases that were operated on due to inguinal hernia, $100 \%$ correspond to the male sex, the dominant age was range of 31 to 59 years with $60 \%$ (3); according to its chronology it has a predominance in $80 \%$ (4) for late recurrence. The technique most used in the first surgical intervention of patients with inguinal hernia was 64\% (38) the Lichtenstein technique and the most used in the first recurrent inguinal hernia surgery included others with $60 \%$ (3). For this reason, the results obtained were shared with surgeons, medical students, the National Hospital of Chiquimula Direction and patients admitted postoperative inguinal hernia.

Keywords: inguinal hernia, recurrence of inguinal hernia, surgical technique 


\section{Introducción}

La corrección de hernias inguinales es un procedimiento quirúrgico que se realiza con más frecuencia en los servicios de cirugía general. De acuerdo con datos del National Center for Health Stadistics, la hernioplastia encabeza la lista de las cinco operaciones quirúrgicas mayores que se han realizado durante varios años (Chinchilla, 2007). Independientemente del número exacto de hernioplastias realizadas, es evidente que constituye un problema de gran magnitud, sobre todo las recurrencias. Cuando la hernia experimenta recurrencia meses o años más tarde, es difícil atribuir el problema a un error quirúrgico. Se ha visto que la recurrencia de la hernia inguinal según la OMS va del 1 al 17\% más frecuente después de hernioplastia primaria de Bassini la cual va del $6 \%$ al $7.3 \%$, siendo más confiable la técnica de Shouldice con $0.6-2.6 \%$ de recurrencias (Barrera, 2015).

La gran diversidad de posibilidades que hay para el manejo de la hernia inguinal, hace que la escogencia de un tipo específico de procedimiento quirúrgico sea muy variable y esté ligado más a una preferencia personal y no a criterios relacionados con el paciente y a los mejores resultados posibles (Palacio, 2016). El siguiente estudio trata de determinar la recurrencia de hernias inguinales y las técnicas de corrección quirúrgica en pacientes mayores de 18 años durante periodo de enero 2013 a diciembre de 2017, en el Hospital Nacional de Chiquimula en los servicios del departamento de cirugía general de adultos con un total de 59 casos que tuvieron tratamiento quirúrgico.

\section{Materiales y métodos}

Estudio descriptivo - retrospectivo. Se revisaron los 59 (100\%) expedientes clínicos de los pacientes que acudieron al Hospital Nacional de Chiquimula y fueron ingresados a los servicios de cirugía general de adultos con diagnóstico de hernia inguinal, durante enero de 2013 a diciembre de 2017. Se incluyeron los expedientes de hombres y mujeres mayores de 18 años con datos de recurrencia de hernia inguinal.

Se recolectaron los datos con base a la boleta diseñada específicamente para el estudio. La boleta se divide por tres series, la primera consta de datos generales del paciente como número de boleta, número de registro del expediente clínico, año, edad, género y procedencia, segunda serie incluyó los tipos de hernias inguinal, localización anatómica y tipo de cirugía; tercera serie incluyó técnica quirúrgica y dispositivos utilizados en la segunda y tercera intervención como su tiempo de evolución en hernias inguinales recurrentes. Se tabularon los datos, se procedió a realizar las tabulaciones, la evaluación estadística y la realización del análisis correspondiente.

\section{Resultados}

\section{Género de pacientes con recurrencia de hernia inguinal}

El total de muestra fueron 5 casos de los cuales se demuestra que el 100\% corresponden al sexo masculino quienes presentaron recurrencia de hernia inguinal. 
Edad de los pacientes con recurrencia de hernias inguinales

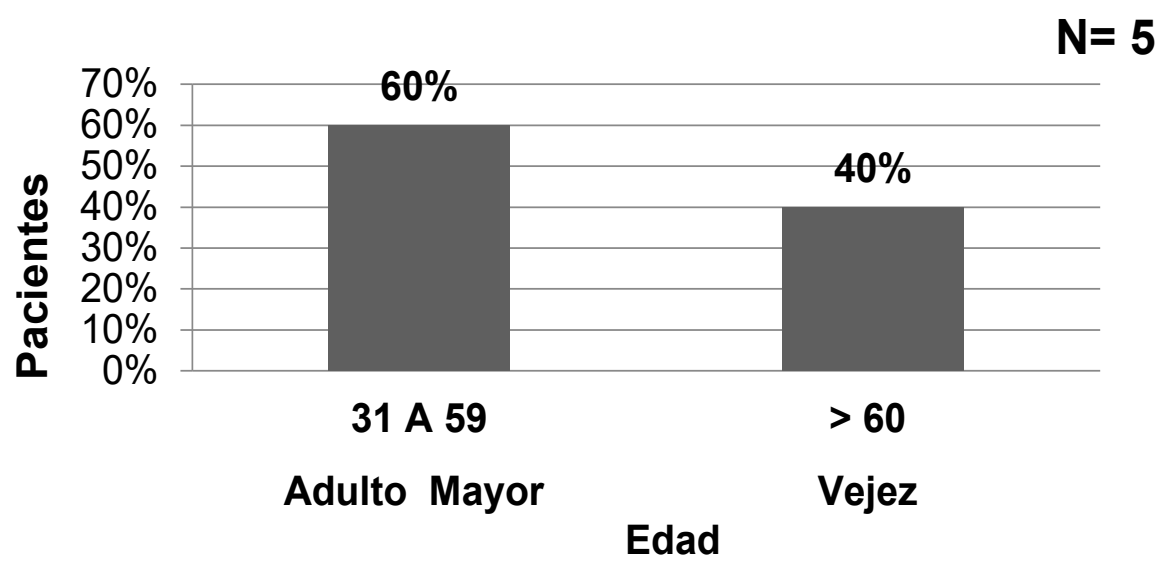

Figura 1. Distribución de pacientes por edad con recurrencia de hernias inguinales atendidas en el servicio de cirugía general de adultos del Hospital Nacional de Chiquimula durante enero de 2013 a diciembre 2017.

Del total de pacientes incluidos en el estudio, la distribución del rango por edades fue de la siguiente manera: con el 60\% (3) de los pacientes se encuentran en el rango de 31 a 59 años, correspondiente según OMS (Organización Mundial de la Salud) a categoría de adulto mayor y con el 40\% (2) en el rango de $>60$ años, correspondiente a la categoría de vejez.

Prevalencia de la recurrencia de hernias inguinales

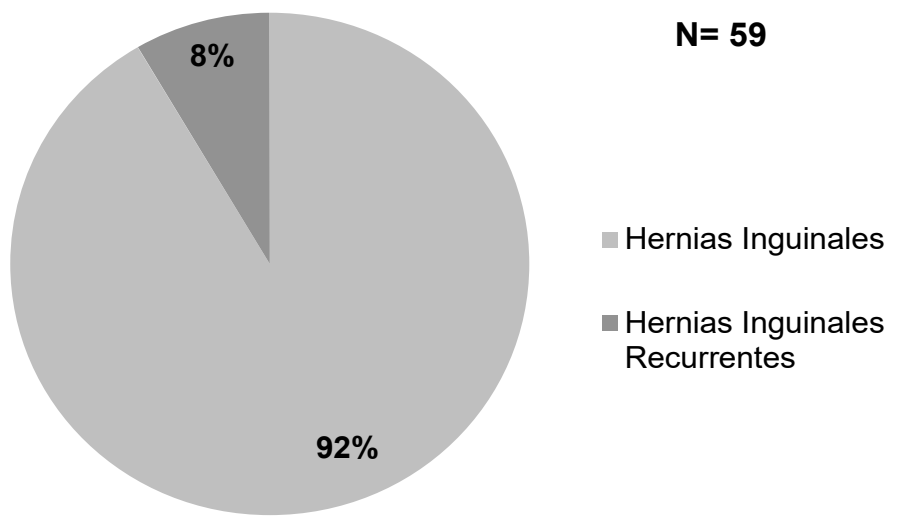

Figura 2. Distribución de la prevalencia de la recurrencia de hernias inguinales en pacientes atendidos en el servicio de cirugía general de adultos del Hospital Nacional de Chiquimula durante enero 2013 a diciembre de 2017.

Del total de la población que fue investigada un 92\% (54) pacientes fueron atendidos para tratamiento de hernia inguinal y una prevalencia de hernia inguinal recurrente del $8 \%$ que corresponde a 5 casos. 
Cronología de la recurrencia de hernias inguinales

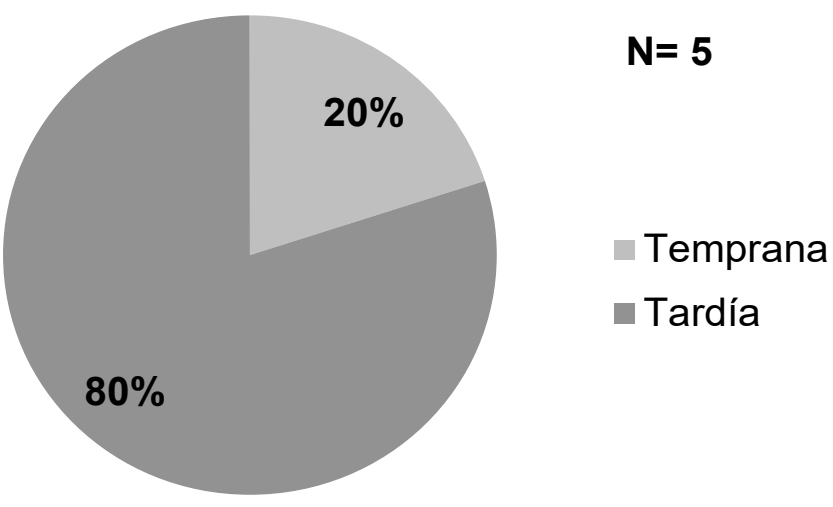

Figura 3. Distribución de la cronología de la recurrencia de hernias inguinales de pacientes atendidos en el servicio de cirugía general de adultos del Hospital Nacional de Chiquimula durante enero 2013 a diciembre 2017.

La cronología de la recurrencia de hernia inguinal tiene predominio en los casos estudiados un $80 \%$ (4) para recurrencia tardía y con un $20 \%$ (1) de casos con recurrencia temprana.

Recurrencia de hernia inguinal según escala de Campanelli

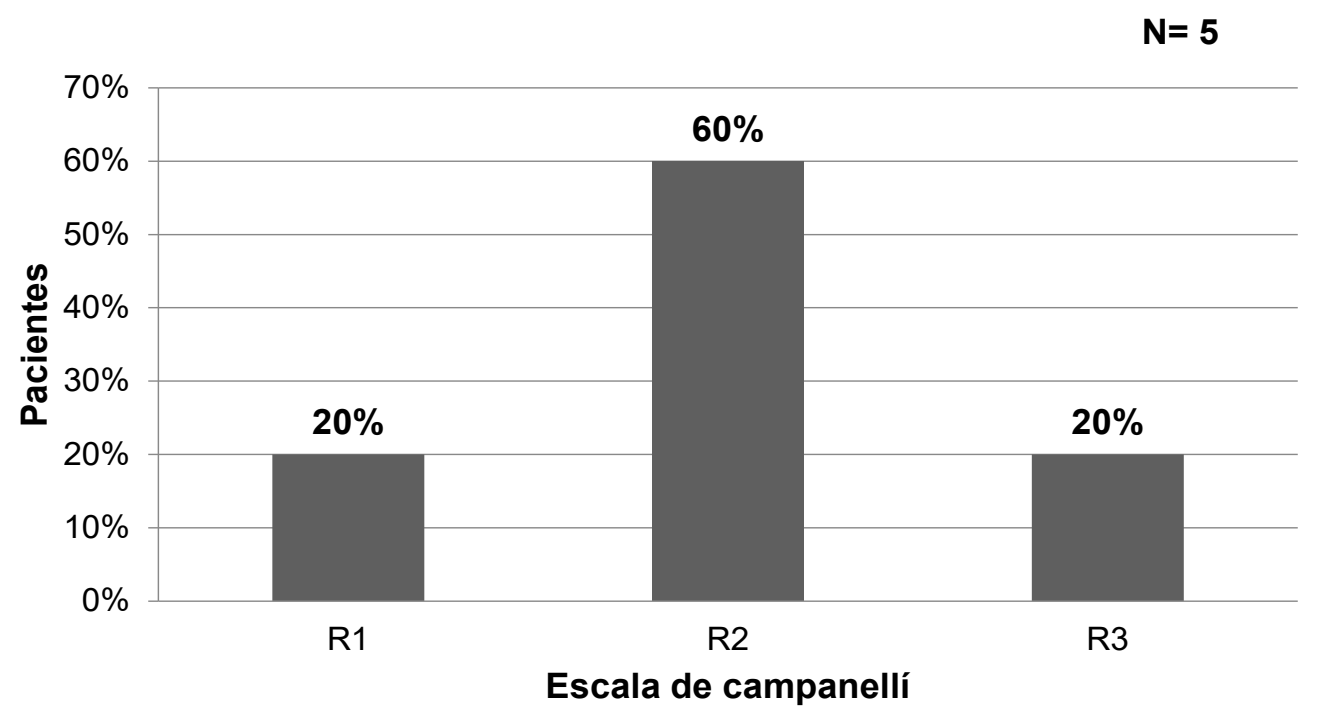

Figura 4. Distribución de la recurrencia de hernias inguinales según escala de Campanelli en pacientes atendidos en el servicio de cirugía general de adultos del Hospital Nacional de Chiquimula durante enero 2013 a diciembre 2017.

La distribución de la siguiente gráfica según escala de Campanelli presenta un predominio de R2 con un total de 60\% (9) de pacientes pertenecientes a primera recidiva de hernia inguinal directa. En igualdad de casos se encuentra R1 con un 20\% (1) de pacientes correspondientes a primera recidiva de hernia inguinal indirecta y R3 con un 20\% (1) de pacientes pertenecientes a todas las hernias inguinales recidivadas más de una vez. 
Clasificación de la hernia inguinal recurrente según escala de Nyhus

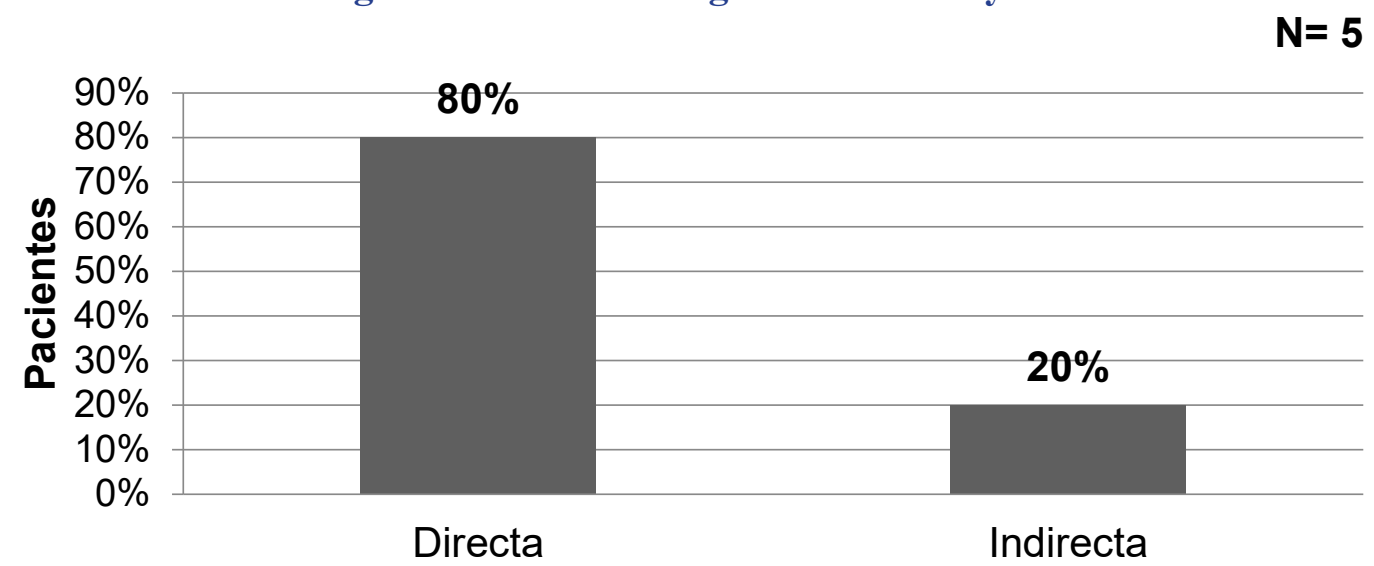

\section{Escala de nyhus}

Figura 5. Distribución de la clasificación de la hernia inguinal recurrente según Escala de Nyhus en pacientes atendidos en el servicio de cirugía general de adultos del Hospital Nacional de Chiquimula durante enero 2013 a diciembre 2017.

De acuerdo a la siguiente distribución de la clasificación según la escala de Nyhus el $80 \%$ (4) de los pacientes tuvo hernia inguinal recurrente tipo directa y el $20 \%$ (1) de los pacientes correspondes a hernia inguinal recurrente tipo indirecta.

\section{Localización anatómica de la hernia inguinal recurrente}

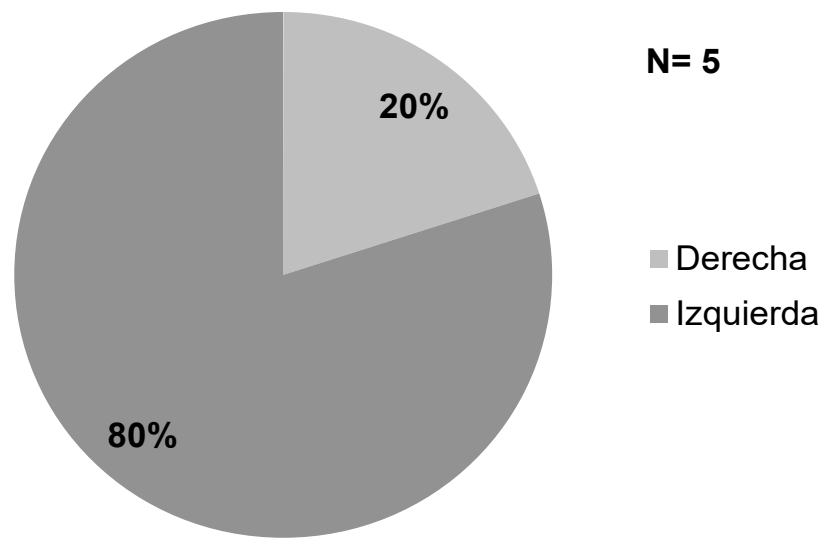

Figura 6. Distribución de la localización anatómica de la hernia inguinal recurrente en pacientes atendidos en el servicio de cirugía general de adultos del Hospital Nacional de Chiquimula durante enero 2013 a diciembre 2017.

La localización anatómica frecuente que se presentó en pacientes con hernia inguinal recurrente fue lado izquierdo con un total de $80 \%$ (4) casos y con el $20 \%$ (1) caso encuentran hernias recurrentes lado derecho. 
Técnica quirúrgica más utilizada en hernias inguinales

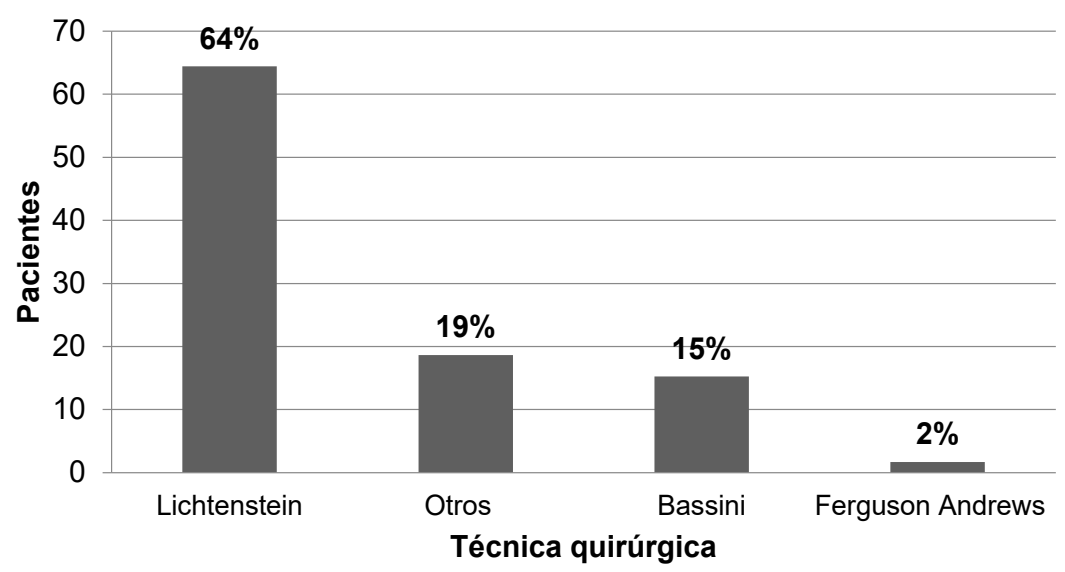

Figura 7. Distribución de la técnica más utilizada en la primera intervención quirúrgica de las hernias inguinales en pacientes atendidos en el servicio de cirugía general de adultos del Hospital Nacional de Chiquimula durante enero 2013 a diciembre 2017.

De acuerdo a la siguiente distribución de la técnica más utilizada en la primera intervención quirúrgica del total de pacientes atendidos con hernia inguinal que corresponde a 59 pacientes en total, de los cuales se encuentra en primer lugar con un 64\% (38) casos técnica de Lichtenstein, segundo lugar con un $19 \%$ (11) casos con otras técnicas, tercer lugar con un 15\% (9) casos técnica de Bassini y por último con un 2\% (1) casos técnica de Ferguson Andrews.

Técnica quirúrgica más utilizada en hernias inguinales recurrentes

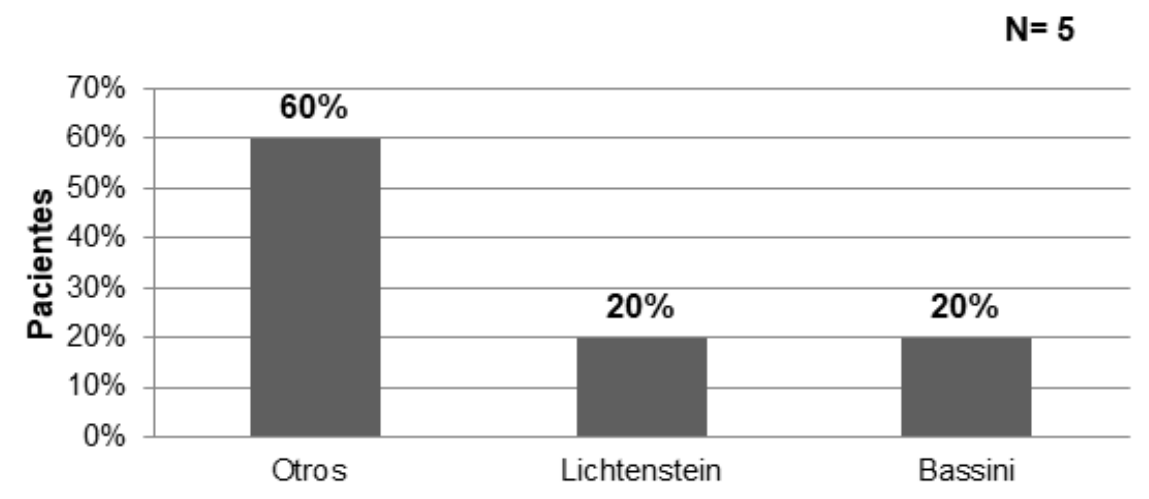

Técnica quirúrgica

Figura 8. Distribución de la técnica más utilizada en la intervención quirúrgica de hernia inguinal recurrente en pacientes atendidos en el servicio de cirugía general de adultos del Hospital Nacional de Chiquimula durante enero 2013 a diciembre 2017.

De acuerdo a la siguiente distribución de la técnica más utilizada en la intervención quirúrgica del total de 5 pacientes con hernia inguinal recurrente, se encuentra en primer lugar técnica que incluye otras con $60 \%$ (3), segundo lugar con 20\%(1) técnica de Lichtenstein y en tercer lugar técnica de Bassini con $20 \%$ (1) caso. 
Tipo de cirugía en las hernias inguinales recurrentes

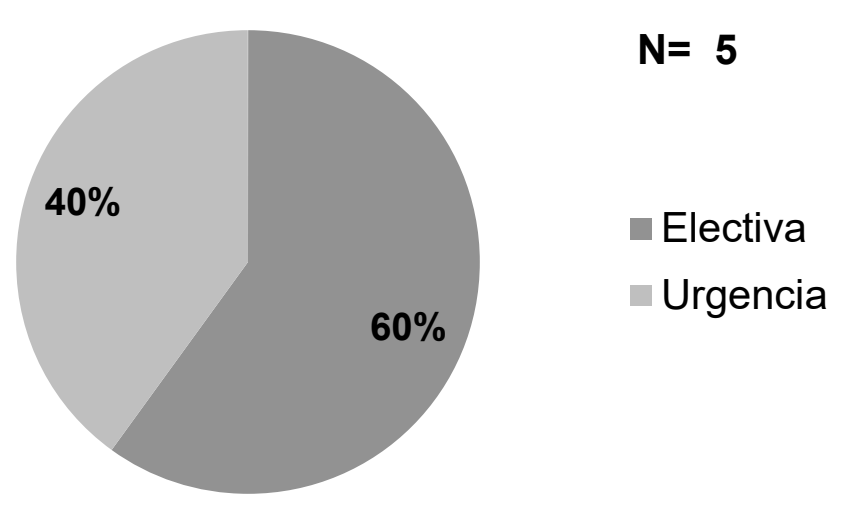

Figura 9. Distribución del tipo de cirugía en la hernia inguinal recurrente en pacientes atendidos en el servicio de cirugía general de adultos del Hospital Nacional de Chiquimula durante enero 2013 a diciembre 2017.

En la siguiente distribución se representa el tipo de cirugía de hernias inguinales recurrentes el total de 5 pacientes atendidos de los cuales el 60\% (3) casos fueron intervenidos de forma electiva y el 40\% (2) casos fueron intervenidos de forma urgente.

\section{Discusión}

El estudio fue realizado con el propósito de determinar la recurrencia de hernia inguinal, en pacientes mayores de 18 años que fueron atendidos en los servicios de cirugía general de adultos del Hospital Nacional de Chiquimula durante enero de 2013 a diciembre de 2017. Se encontró que, de 59 casos que recibieron tratamiento quirúrgico de hernioplastia y/o herniorrafia, se obtuvo una prevalencia total del $8 \%$ (5) casos que tuvieron recurrencia, quedando aun dentro de los rangos que la OMS tiene para la recurrencia hernia inguinal que van del 1-17\%. Demostrando que el predominio de la recurrencia de hernia inguinal fue el sexo masculino con un total del 100\% de casos. Del total de pacientes incluidos en el estudio, la distribución del rango por edades fue de la siguiente manera: con el 60\% (3) de los pacientes se encuentran en el rango de 31 a 59 años, correspondiente según OMS a categoría de adulto mayor y con el 40\% (2) en el rango de > 60 años, correspondiente a la categoría de vejez, sin ningún caso encontrado entre las edades de adolescencia tardía y adulto joven.

En cuanto a la cronología de la recurrencia de hernia inguinal tiene predominio en los casos estudiados un $80 \%$ (4) para recurrencia tardía, que equivale a una aparición después de tres años tras el tratamiento quirúrgico primario y con un $20 \%$ (1) de casos con recurrencia temprana, que equivale a la aparición de la recurrencia de hernia inguinal antes de los tres años, quedando dentro del rango que resalta la revista Universidad y Ciencia de la ciudad de México en su estudio Recurrencia de Hernias Inguinales durante año 2016, que indica dicha aparición tardía se pueda deber a factores propiamente del paciente y no asociados a técnica quirúrgica. 
La escala de Campanelli implementada desde el año 1996 ha sido de referencia para clasificación de hernias inguinales recidivantes o recurrentes la cual va desde tipo R1 a R3 respectivamente, según resultados de éste estudio en base a la clasificación se encuentra que hay un predominio de R2 con un total de $60 \%$ (3) de pacientes, pertenecientes a primera recidiva de hernia inguinal tipo directa y en igualdad de casos se encuentra R1 con un 20\% (1) de pacientes correspondientes a primera recidiva de hernia inguinal tipo indirecta y R3 con un 20\% (1) de pacientes pertenecientes a todas las hernias inguinales recidivadas más de una vez.

Tomando en cuenta la clasificación según la escala de Nyhus el 80\% (4) de los pacientes tuvo hernia inguinal recurrente tipo directa y el $20 \%$ (1) de los pacientes correspondes a hernia inguinal recurrente tipo indirecta y ningún paciente con hernia inguinal recurrente se encontró con el tipo combinada. La localización anatómica frecuente que se presentó en pacientes con hernia inguinal recurrente fue lado izquierdo con un total de 4 casos que equivale a un $80 \%$ y en segundo lugar con 1 caso equivalente al $20 \%$ se encuentran lado derecho; sin encontrarse hernias recurrentes bilaterales.

Se observó que de la técnica más utilizada en la primera intervención quirúrgica del total de pacientes atendidos con hernia inguinal que correspondieron a 59 casos se tiene en primer lugar con un 64\% (38) casos la técnica utilizada con dispositivos fue la de Lichtenstein, segundo lugar con un 19\% (11) casos con otras técnicas entre las cuales se encontró tipo Harkins, tercer lugar con un 15\% (9) casos técnica de Bassini que es una de las técnicas estudiadas que han demostrado mayor recurrencia de hernia inguinal según el estudio de la Universidad y Ciencia de la Ciudad de México y por último con un 2\% (1) casos técnica de Ferguson Andrews y ningún caso encontrado con la técnica de Shouldice.

En cuanto a la reparación de hernias inguinales recurrentes la técnica más utilizada se tiene en primer lugar la categoría de otros que incluye por ejemplo la técnica quirúrgica Harkins y sus variaciones con $60 \%$ (3) que son técnicas con tensión, en segundo lugar, se encuentra la técnica de Lichtenstein con $20 \%$ (1) y en tercer lugar la técnica de Bassini con 20\% (1). Aumentando así la probabilidad que la recurrencia continúe, ya que la única técnica sin tensión de la cual se ha demostrado una reducción del 50 a 75\% de la recurrencia de hernia inguinal es la tipo Lichtenstein; según el estudio reparación abierta de hernia inguinal: una revisión basada en pruebas de la Revista de Clínicas Quirúrgicas de Norteamérica publicada en el año 2008.

Además se logró evidenciar que la mayoría de hernioplastias efectuadas con un $60 \%$ (3) casos fueron intervenidos de forma electiva y el 40\% (2) casos fueron intervenidos de forma urgente, comparado con el estudio "Comportamiento de las hernias inguinales y su recurrencia en el servicio de cirugía del Hospital Nacional de Chiquimula en el año 2007" en el cual la mayoría siguen siendo de reparación electiva, queda demostrado que el tipo de cirugía no ha influido en la recurrencia de las mismas.

\section{Agradecimientos}

Al Hospital Nacional de Chiquimula, en especial al Comité de Docencia e Investigación por la aprobación para realizar la investigación. Un agradecimiento especial a los doctores Graciela Icaza, Edvin Danilo Mazariegos Albanés y Carlos Arriola Monasterio, por ser unos excelentes guías y apoyo durante la realización de la investigación. 


\section{Referencias bibliográficas}

Barrera, W. (2015). Manejo de pacientes con hernia inguinal recidivante (en línea). Tesis M.Sc. Guatemala, USAC. p. 41. Disponible en http://www.repositorio.usac.edu.gt/4340/1/WENCESLAO\% 20BARRERA\%20GODOY.pdf

Chinchilla, L. (2007). Comportamiento de las hernias inguinales y su recurrencia en pacientes de cirugía General del Hospital Modular de Chiquimula. Chiquimula, Guatemala, USAC-CUNORI.

Palacio, A. (2016). Epidemiología y factores que influyen en la recidiva de pacientes intervenidos por hernia inguinal en una Institución de nivel II de la ciudad de Bogotá, 2005.2014. Universidad Nacional de Colombia. P.41 Consultado 12 feb. 2018. Disponible en http://bdigital.unal.edu. co/55766/1/1053771162.2016.pdf

\section{Sobre la autora}

\section{Danna Aby Danelys Morales Carías}

Médico y Cirujano, egresada del Centro Universitario de Oriente, CUNORI, de la Universidad de San Carlos de Guatemala. En el año 2018 realizó su proyecto de investigación con el título "Recurrencia de hernias inguinales en el Hospital de Chiquimula".

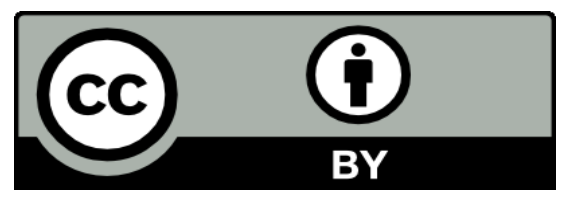

Este texto está protegido por una licencia CreativeCommons 4.0.

Usted es libre para compartir, copiar y redistribuir el material en cualquier medio o formato y adaptar el documento, remezclar, transformar y crear a partir del material para cualquier propósito, incluso comercialmente, siempre que cumpla la condición de atribución: usted debe reconocer el crédito de una obra de manera adecuada, proporcionar un enlace a la licencia, e indicar si se han realizado cambios. Puede hacerlo en cualquier forma razonable, pero no de forma tal que sugiera que tiene el apoyo del licenciante o lo recibe por el uso que hace. 